\title{
JORDAN DECOMPOSITION OF BILINEAR FORMS
}

\author{
DragomiR Ž. ĐOKOvić AND KAIMING ZHAO
}

\begin{abstract}
Let $V$ be an $n$-dimensional vector space over an algebraically closed field $K$ of characteristic 0 . Denote by $\mathcal{B}$ the space of bilinear forms $f: V \times V \rightarrow K$. We say that $g \in \mathcal{B}$ is semisimple if the orbit $\mathcal{O}_{g}=\mathrm{SL}_{n} \cdot g$ is closed in $\mathcal{B}$, in the Zariski topology. We say that $h \in \mathcal{B}$ is a null-form if $0 \in \overline{\mathcal{O}_{h}}$, the Zariski closure of $\mathcal{O}_{h}$. We introduce the Jordan decomposition for bilinear forms $f=g+h(g$ semisimple, $h$ a null-form) in analogy with the well known Jordan decomposition for linear operators. While the latter decomposition is unique, this is not the case for the former. If $f$ is not a null-form, we introduce the primary decomposition of $f$ and use it to construct all possible Jordan decompositions of $f$.
\end{abstract}

\section{Introduction}

It is well known that any linear operator on a finite dimensional vector space over a perfect field can be uniquely decomposed into a sum of a semisimple operator and a nilpotent operator which commute. This decomposition is known as Jordan decomposition. In this paper we are going to introduce similar decomposition for bilinear forms.

We work over an algebraically closed field $K$ of characteristic 0 . Fix an $n$ dimensional vector space $V$ over $K$ and denote by $\mathcal{B}$ the space of bilinear forms $f: V \times V \rightarrow K$. We shall use the natural action of $\mathrm{GL}_{n}:=\mathrm{GL}(V)$ and its subgroup $\mathrm{SL}_{n}:=\mathrm{SL}(V)$ on $\mathcal{B}$.

We say that $f \in \mathcal{B}$ is semisimple if the orbit $\mathcal{O}_{f}:=\mathrm{SL}_{n} \cdot f$ is closed in $\mathcal{B}$. Let $K[\mathcal{B}]$ denote the algebra of polynomial functions on $\mathcal{B}$. The categorical quotient $\mathcal{B} / / \mathrm{SL}_{n}$, which parametrizes the closed $\mathrm{SL}_{n}$-orbits in $\mathcal{B}$, is the affine variety associated with the subalgebra $K[\mathcal{B}]^{\mathrm{SL}_{n}}$ of $\mathrm{SL}_{n}$-invariants in $K[\mathcal{B}]$. In this case, this quotient is an affine space (see the next section). The inclusion map $K[\mathcal{B}]^{\mathrm{SL}_{n}} \rightarrow K[\mathcal{B}]$ gives rise to the canonical projection $\pi: \mathcal{B} \rightarrow \mathcal{B} / / \mathrm{SL}_{n}$, which is known to be surjective [8, Theorem 4.6].

Let $\mathcal{F}(f)$ denote the fiber of $\pi$ over the point $\pi(f) \in \mathcal{B} / / \mathrm{SL}_{n}$, i.e., $\mathcal{F}(f):=$ $\pi^{-1}(\pi(f))$. The zero fiber $\mathcal{F}(0)$ is known as the Hilbert null-cone for the action $\left(\mathrm{SL}_{n}, \mathcal{B}\right)$ and will be denoted by $\mathcal{N}$. We shall refer to the bilinear forms $f \in \mathcal{N}$ as the null-forms. If $f \in \mathcal{B}$, we recall (see e.g. [8, p. 157]) that $f \in \mathcal{N}$ iff $0 \in \overline{\mathcal{O}_{f}}$, where $\overline{\mathcal{O}_{f}}$ is the Zariski closure of $\mathcal{O}_{f}$.

Received October 1, 2004.

2000 Mathematics Subject Classification. 14L35, 14L24, 20G05.

The first author was supported in part by the NSERC Grant A-5285 and the second by the NSF of China (Grant 10371120 and 10431040) and the NSERC. 
Any fiber $\mathcal{F}(f)$ contains a unique closed $\mathrm{SL}_{n}$-orbit, which we denote by $\mathcal{C}_{f}$. For $g \in \mathcal{C}_{f}$, the isotropy subgroups $Z(g)=\left(\mathrm{GL}_{n}\right)_{g}$ and $\left(\mathrm{SL}_{n}\right)_{g}$ are reductive. As a $Z(g)$-module, $\mathcal{B}$ decomposes as $\mathcal{B}=T_{g} \oplus N$, where $T_{g}$ is the tangent space of the orbit $\mathcal{C}_{f}=\mathcal{O}_{g}$ at the point $g$ and $N$ is a complementary submodule. Let $\mathcal{N}_{g}$ denote the Hilbert null-cone for the induced action of $\left(\mathrm{SL}_{n}\right)_{g}$ on $N$ (see $[8$, $\S 5]$ ). Observe that $\mathcal{N}_{g} \subset \mathcal{N}$ and that $\mathcal{N}_{g}$ depends on the choice of $N$.

Any $f \in \mathcal{B}$ can be decomposed as a sum of a semisimple bilinear form $g \in \mathcal{C}_{f}$ and a null-form $h \in \mathcal{N}_{g}$, but this decomposition is not unique due to the nonuniqueness of $N$ (see [8, p. 213]). Such decomposition $f=g+h$ we will call a Jordan decomposition if the additional condition: $\left(\mathrm{GL}_{n}\right)_{f}=\left(\mathrm{GL}_{n}\right)_{g} \cap\left(\mathrm{GL}_{n}\right)_{h}$ is satisfied. The Jordan decomposition is not unique in general, but we are able to describe and construct all such decompositions.

A posteriori, it turns out that there is a link between the two kind of Jordan decompositions: one for linear operators and the other for bilinear forms. This link is visible in the case of nondegenerate bilinear forms. Thus assume that $f \in \mathcal{B}$ is nondegenerate. Its asymmetry is the unique linear operator $\sigma$ such that $f(y, x)=f(x, \sigma(y))$ for all $x, y \in V$. If $f=g+h$ is a Jordan decomposition of $f$, as above, then $g$ is also nondegenerate and its asymmetry is equal to the semisimple component of the asymmetry $\sigma$ of $f$ (in its Jordan decomposition).

In Section 2 we state the Canonical Form Theorem 2.1 for bilinear forms in our context. This theorem has been known for long time apart from the explicit canonical forms given in part (b), which have been constructed very recently [6]. We also give an explicit formula for the canonical projection $\pi: \mathcal{B} \rightarrow \mathcal{B} / / \mathrm{SL}_{n}$.

In Section 3 we characterize the semisimple bilinear forms as well as the nullforms in terms of their indecomposable summands. We also show how to find, for a given $f \in \mathcal{B}$, a representative of the unique closed orbit $\mathcal{C}_{f}$ contained in the fiber $\mathcal{F}(f)$. Finally, we define the primary decomposition

$$
(V, f)=\perp_{\hat{\lambda} \in \hat{K}}\left(V^{\hat{\lambda}}, f^{\hat{\lambda}}\right)
$$

of a bilinear space $(V, f)$. The primary components $\left(V^{\hat{\lambda}}, f^{\hat{\lambda}}\right)$ are parametrized by the subsets $\hat{\lambda}$ of $K$ which are either singletons $\{\lambda\}$ if $\lambda=0, \pm 1$ or two-element subsets $\left\{\lambda, \lambda^{-1}\right\}$ if $\lambda \neq 0, \pm 1$. The case when $f$ is nondegenerate was treated in [9] in more generality.

In Section 4 we define the Jordan decomposition for bilinear forms $f=$ $f_{\mathrm{ss}}+f_{\mathrm{nl}}$, with $f_{\mathrm{ss}}$ semisimple and $f_{\mathrm{nl}}$ a null-form, and discuss some of its properties. The main result of this section (Theorems 4.5 and 4.6) shows that the construction of Jordan decomposition reduces (in a straightforward manner) to the same problem in the primary case. As mentioned in the abstract, the Jordan decomposition of bilinear forms is not unique (in general). However, if $f$ is semisimple resp. a null-form then $f_{\mathrm{ss}}=f, f_{\mathrm{nl}}=0$ resp. $f_{\mathrm{ss}}=0, f_{\mathrm{nl}}=f$ is the unique Jordan decomposition of $f$.

In Section 5 we treat the primary case: $(V, f)=\left(V^{\hat{\lambda}}, f^{\hat{\lambda}}\right)$. If $\lambda \neq \pm 1$ and $f$ is not semisimple, then there are infinitely many Jordan decompositions of $f$. These decompositions $f=f_{\mathrm{ss}}+f_{\mathrm{nl}}$ are parametrized by a scalar $\mu \in K \cup$ 
$\{\infty\}, \mu \neq \lambda$. The null-form component $f_{\mathrm{nl}}$ is symmetric resp. skew-symmetric precisely when $\mu=1$ resp. $\mu=-1$. Unexpectedly, it turned out that in the cases $\lambda= \pm 1$ the Jordan decomposition is unique. In fact, $f_{\mathrm{ss}}=f^{+}, f_{\mathrm{nl}}=f^{-}$ if $\lambda=1$ and $f_{\mathrm{ss}}=f^{-}, f_{\mathrm{nl}}=f^{+}$if $\lambda=-1$. (By $f^{+}$resp. $f^{-}$we denote the symmetric resp. skew-symmetric component of $f$.)

As far as we know, the concept of Jordan decomposition in the general setting of linear algebraic groups was introduced first by V. Kac and E.B. Vinberg in their paper [7]. In an important paper [10], Spaltenstein has constructed for bilinear forms a particular Jordan decomposition in the sense of Kac and Vinberg. The definition that we have adopted is also in agreement with Kac and Vinberg definition but is more demanding. As a consequence, the Jordan decomposition constructed by Spaltenstein, using an ad hoc method, is not a Jordan decomposition in our sense.

\section{Preliminaries}

For $f \in \mathcal{B}$, we refer to $(V, f)$ as a bilinear space. If $f$ is fixed or known from the context, we may say that $V$ itself is a bilinear space.

The transpose of a bilinear form $f: V \times V \rightarrow K$ is the bilinear form $g$ : $V \times V \rightarrow K$ such that $g(x, y)=f(y, x)$ for all $x, y \in V$. We shall denote the transpose of $f$ by $f^{\prime}$.

We have $\mathcal{B}=\mathcal{B}^{+} \oplus \mathcal{B}^{-}$where $\mathcal{B}^{+}=\left\{f \in \mathcal{B}: f^{\prime}=f\right\}$ is the space of symmetric forms and $\mathcal{B}^{-}=\left\{f \in \mathcal{B}: f^{\prime}=-f\right\}$ that of skew-symmetric forms. For $f \in \mathcal{B}$ we have $f=f^{+}+f^{-}$where

$$
f^{+}=\frac{1}{2}\left(f+f^{\prime}\right) \in \mathcal{B}^{+}, \quad f^{-}=\frac{1}{2}\left(f-f^{\prime}\right) \in \mathcal{B}^{-} .
$$

If $V_{1}$ and $V_{2}$ are subspaces of $V$ and $f\left(V_{1}, V_{2}\right)=0=f\left(V_{2}, V_{1}\right)$, we say that $V_{1}$ and $V_{2}$ are orthogonal to each other with respect to $f$, and we write $V_{1} \perp V_{2}$. If also $V=V_{1} \oplus V_{2}$, then we write $V=V_{1} \perp V_{2}$ and $f=f_{1} \perp f_{2}$ where $f_{1}=\left.f\right|_{V_{1} \times V_{1}}, f_{2}=\left.f\right|_{V_{2} \times V_{2}}$. We shall also express this situation by writing

$$
(V, f)=\left(V_{1}, f_{1}\right) \perp\left(V_{2}, f_{2}\right)
$$

and we say that $\left(V_{1}, f_{1}\right)$ and $\left(V_{2}, f_{2}\right)$ are summands of $(V, f)$.

We say that $(V, f)$ or $f$ is decomposable if $(V, f)$ has a nonzero proper summand. We say that $(V, f)$ or $f$ is indecomposable if $V \neq 0$ and $(V, f)$ is not decomposable.

We fix a basis $\alpha=\left\{v_{1}, v_{2}, \ldots, v_{n}\right\}$ of $V$. Let $f \in \mathcal{B}$. The $n$-by- $n$ matrix $A_{f}^{\alpha}=\left(a_{i j}\right)$, with $a_{i j}=f\left(v_{i}, v_{j}\right)$, is the matrix of $f$ with respect to this basis. We denote the transpose of any matrix $X$ by $X^{\prime}$. The matrix of $f^{\prime}$, with respect to the same basis, is $\left(A_{f}^{\alpha}\right)^{\prime}$, i.e., $A_{f^{\prime}}^{\alpha}=\left(A_{f}^{\alpha}\right)^{\prime}$. Later we shall simply write $A_{f}=A_{f}^{\alpha}$, or $f=A_{f}$ (if the basis is chosen).

If $\beta$ is another basis of $V$, we know that $A_{f}^{\alpha}$ and $A_{f}^{\beta}$ are congruent, i.e., there exists $P \in \mathrm{GL}_{n}(K)$ such that $A_{f}^{\beta}=P^{\prime} A_{f}^{\alpha} P$. 
Let us consider a bilinear space $(V, f)$ and a subspace $U \subset V$. Then we define the subspaces

$$
L(U):=\{v \in V: f(v, U)=0\}
$$

and

$$
R(U):=\{v \in V: f(U, v)=0\} .
$$

If we need to indicate $f$, we shall write $L_{f}$ and $R_{f}$ instead of $L$ and $R$, respectively.

Some interesting properties of the operators $L$ and $R$ were established in [4]. We shall recall a few of them later when needed. We say that a subspace $U$ is totally isotropic if $f(U, U)=0$, i.e., $U \subset L(U)$ (or $U \subset R(U)$ ).

We say that $f \in \mathcal{B}$ is nondegenerate resp. degenerate if its matrix $A_{f}$ is nonsingular resp. singular. This is equivalent to the condition $L(V)=0$ (or $R(V)=0$ ). A bilinear space is totally degenerate if it has no nonzero nondegenerate summands.

We shall denote the (lower triangular) Jordan block of order $m$ with eigenvalue $\lambda \in K$ by $J_{m}(\lambda)$. For convenience, we define $n$-by- $n$ matrices $H_{n}(\lambda)$ and $\Gamma_{n}$ by

$$
H_{n}(\lambda)=\left[\begin{array}{cc}
0 & I_{m} \\
J_{m}(\lambda) & 0
\end{array}\right], \quad n=2 m, \lambda \in K
$$

and

$$
\Gamma_{n}=\left[\begin{array}{rrrrrrcc}
0 & 0 & 0 & 0 & \cdots & 0 & 0 & (-1)^{n-1} \\
0 & 0 & 0 & 0 & \cdots & 0 & (-1)^{n-2} & (-1)^{n-2} \\
\vdots & & & & & & & \\
0 & -1 & -1 & 0 & \cdots & 0 & 0 & 0 \\
1 & 1 & 0 & 0 & \cdots & 0 & 0 & 0
\end{array}\right]
$$

Two bilinear forms $f: V \times V \rightarrow K$ and $g: W \times W \rightarrow K$ are equivalent if there exists a vector space isomorphism $\varphi: V \rightarrow W$ such that $g(\varphi(x), \varphi(y))=f(x, y)$, $\forall x, y \in V$.

Now we can state the Canonical Form Theorem for bilinear forms (see [6] and, for an older version, [2]).

Theorem 2.1. (a) Any $f \in \mathcal{B}$ admits an orthogonal direct decomposition

$$
f=f_{1} \perp f_{2} \perp \cdots \perp f_{k},
$$

where the $f_{i}$ 's are indecomposable bilinear forms which are unique up to equivalence and permutation.

(b) If $f \in \mathcal{B}$ is indecomposable, then we can find a basis $\alpha$ for $V$ such that $A_{f}^{\alpha}$ is one of the following matrices:

(b1) $H_{n}(\lambda), n=2 m, \lambda \neq(-1)^{m+1}$;

(b2) $\Gamma_{n}, n \geq 1$;

(b3) $J_{n}(0), n=2 m+1$. 
(c) The matrices listed in part (b) are pairwise non-congruent except for the fact that $H_{n}(\lambda)$ and $H_{n}\left(\lambda^{-1}\right)$ are congruent when $\lambda \neq 0, \pm 1$.

We mention that, when $n=2 m$ is even, $H_{n}(0)$ is congruent to $J_{n}(0)$.

Next we recall some pertinent results from $[3,10]$ concerning the geometry of $\mathrm{SL}_{n}$-orbits in $\mathcal{B}$. The natural (congruence) action of $\mathrm{GL}_{n}$ on $\mathcal{B}$ is defined by $(a, f) \rightarrow a \cdot f$, where $a \in \mathrm{GL}_{n}, f \in \mathcal{B}$, and $a \cdot f \in \mathcal{B}$ is given by

$$
(a \cdot f)(x, y)=f\left(a^{-1}(x), a^{-1}(y)\right), \quad \forall x, y \in V .
$$

The subspaces $\mathcal{B}^{+}$and $\mathcal{B}^{-}$are $\mathrm{GL}_{n}$-submodules of $\mathcal{B}$ (both simple if $n \geq 2$ ). The stabilizer of a point $f \in \mathcal{B}$ in a subgroup $G$ of $\mathrm{GL}_{n}$ will be denoted by $G_{f}$.

Denote the Lie algebras of $\mathrm{GL}_{n}$ and $\mathrm{SL}_{n}$ by $\mathfrak{g l}_{n}$ and $\mathfrak{s l}_{n}$, respectively. By differentiating the action of $\mathrm{GL}_{n}$, we obtain an action $(u, f) \rightarrow u \cdot f$ of $\mathfrak{g l}_{n}$ on $\mathcal{B}$. The bilinear form $u \cdot f$ is given by

$$
(u \cdot f)(x, y)=-f(u(x), y)-f(x, u(y)), \quad x, y \in V .
$$

We set $n=2 m$ if $n$ is even and $n=2 m+1$ if $n$ is odd. For $f \in \mathcal{B}$, we define $\operatorname{det}(f)=\operatorname{det}\left(A_{f}\right)=\left|A_{f}\right|$. This gives a polynomial function det $: \mathcal{B} \rightarrow K$ which is homogeneous of degree $n$. It is independent (up to a scalar factor) of the choice of the basis of $V$. Next define

$$
P(f, t)=\left|A_{f^{+}}-t A_{f^{-}}\right| .
$$

The coefficients of the odd powers of $t$ in the expansion of this determinant are all zero. Hence we have

$$
P(f, t)=P_{0}(f)+P_{1}(f) t^{2}+\cdots+P_{m}(f) t^{2 m},
$$

where $P_{0}, P_{1}, \ldots, P_{m} \in K[\mathcal{B}]$ are homogeneous polynomials of degree $n$. In particular, $P_{0}(f)=\operatorname{det}\left(A_{f^{+}}\right)$and, if $n$ is even, $P_{m}(f)=\operatorname{det}\left(A_{f^{-}}\right)$. Let us extend the Pfaffian function $\operatorname{Pf}: \mathcal{B}^{-} \rightarrow K$ to $\mathcal{B}$ by setting $\operatorname{Pf}(f)=\operatorname{Pf}\left(A_{f^{-}}\right)$for any $f \in \mathcal{B}$. Hence, if $n$ is even, then $P_{m}=\operatorname{Pf}^{2}$.

The following basic result was obtained by A.O. Adamovich and E.O. Golovina [1]. (They have also proved similar results for the $\mathrm{SL}_{n}$-modules $\mathcal{B}^{+} \oplus \mathcal{B}^{+}$ and $\mathcal{B}^{-} \oplus \mathcal{B}^{-}$.)

Theorem 2.2. The algebra $K[\mathcal{B}]^{\mathrm{SL}_{n}}$ is generated by the polynomials $P_{0}, P_{1}$, $\ldots, P_{m}$ if $n$ is odd and by $P_{0}, P_{1}, \ldots, P_{m-1}$ and $\mathrm{Pf}$ if $n$ is even. Moreover these generators are algebraically independent.

By the above theorem, the categorical quotient $\mathcal{B} / / \mathrm{SL}_{n}$ is an affine $(m+1)$ dimensional space over $K$. The canonical projection

$$
\pi: \mathcal{B} \rightarrow \mathcal{B} / / \mathrm{SL}_{n}=K^{m+1}
$$

is defined by

$$
\pi(f):=\left(P_{0}(f), P_{1}(f), \ldots, P_{m-1}(f), P_{m}(f)\right)
$$

if $n$ is odd, and by

$$
\pi(f):=\left(P_{0}(f), P_{1}(f), \ldots, P_{m-1}(f), \operatorname{Pf}(f)\right)
$$


if $n$ is even.

The Hilbert null-cone $\mathcal{N}=\mathcal{F}(0)$ is the set of common zeros in $\mathcal{B}$ of the homogeneous polynomial generators of $K[\mathcal{B}]^{\mathrm{SL}_{n}}$ listed in the above theorem. Thus an element $f \in \mathcal{B}$ belongs to $\mathcal{N}$ iff the polynomial $P(f, t)$, in the variable $t$, is zero.

\section{Primary decomposition of bilinear forms}

The main objective of this section is to introduce the primary decomposition of bilinear spaces $(V, f)$. We define such decomposition only when $f \notin \mathcal{N}$. In the nondegenerate case, this was done already in Riehm's paper [9].

It is well known (see [8, Theorem 4.7, Corollary]) that every fiber $\mathcal{F}(f)$ contains a unique closed $\mathrm{SL}_{n}$-orbit. We denote this orbit by $\mathcal{C}_{f}$. Since $\overline{\mathcal{O}_{f}}$ contains a closed orbit (see e.g. [8, p. 149]), we must have

$$
\mathcal{C}_{f} \subset \overline{\mathcal{O}_{f}} \subset \mathcal{F}(f) .
$$

We define $(-)^{k}$ to be the sign + if $k$ is even and the sign - if $k$ is odd.

Proposition 3.1. If $f \in \mathcal{B}$ is indecomposable (see Theorem 2.1), then a representative $f_{c}$ of $\mathcal{C}_{f}$ is given by:

(b1) $f_{c}=\left[\begin{array}{cc}0 & I_{m} \\ \lambda I_{m} & 0\end{array}\right]$ if $f=H_{n}(\lambda), n=2 m, \lambda \neq(-1)^{m+1}$;

(b2) $f_{c}=f^{(-)^{n+1}}$ if $f=\Gamma_{n}$;

(b3) $f_{c}=0$ if $f=J_{n}(0), n=2 m+1$.

Proof. The bilinear forms $f_{c}$ listed in the proposition are semisimple by [3]. So we need only show that $f_{c} \in \overline{\mathcal{O}_{f}}$ in each case.

(b1) For $t \in K^{*}$ we have

$$
X=\operatorname{diag}\left(1, t^{-1}, t^{-2}, \ldots, t^{1-m}, 1, t, t^{2}, \ldots, t^{m-1}\right) \in \mathrm{SL}_{n} .
$$

Hence $X^{\prime} f X=f_{c}+t\left(f-f_{c}\right)$, and so $f_{c} \in \overline{\mathcal{O}_{f}}$.

(b2) For $t \in K^{*}$ we set

$$
X=\operatorname{diag}\left(t^{-m}, t^{1-m}, \ldots, t^{-1}, 1, t, t^{2}, \ldots, t^{m}\right) \in \mathrm{SL}_{n}
$$

if $n=2 m+1$, and

$$
X=\operatorname{diag}\left(t^{-m}, t^{1-m}, \ldots, t^{-1}, t, t^{2}, \ldots, t^{m}\right) \in \mathrm{SL}_{n}
$$

if $n=2 m$. Then again $X^{\prime} f X=f_{c}+t\left(f-f_{c}\right)$ and $f_{c} \in \overline{\mathcal{O}_{f}}$.

(b3) This holds because $f \in \mathcal{N}$ since $P(f, t)=0$.

Since the semisimple bilinear forms as well as the null-forms play an essential role in this paper, it is important to have simple characterizations of these two types of bilinear forms. The following proposition provides such characterizations. 
Proposition 3.2. Let $f \in \mathcal{B}$ and let $f=f_{1} \perp f_{2} \perp \cdots \perp f_{k}$, where each $f_{i}$ is indecomposable. Then

(a) $f \in \mathcal{N}$ iff at least one $f_{i}$ is of type (b3) (see Theorem 2.1).

(b) For $f \neq 0, f$ is semisimple iff each $f_{i}=\left[\lambda_{i}\right]$ for some $\lambda_{i} \in K^{*}$ or

$$
f_{i}=\left[\begin{array}{cc}
0 & \mu_{i} \\
\nu_{i} & 0
\end{array}\right] \quad \text { with } \quad \mu_{i} \neq \nu_{i}
$$

Proof. The first assertion follows from [3] and the second from the previous proposition.

The following proposition follows immediately from Proposition 3.2 (a) and [4, Theorem $3.2(\mathrm{~d})]$.

Proposition 3.3. For $f \in \mathcal{B} \backslash \mathcal{N}$ there is a unique decomposition

$$
(V, f)=\left(V_{\mathrm{tdeg}}, f_{\mathrm{tdeg}}\right) \perp\left(V_{\text {ndeg }}, f_{\text {ndeg }}\right),
$$

where the first summand is totally degenerate and the second nondegenerate.

We give some more details. The subspaces $L^{2 k+1}(V)\left(\right.$ resp. $\left.L^{2 k}(V)\right)$ are all equal for large $k$ and we denote this subspace by $L^{\text {odd }}(V)$ (resp. $L^{\text {even }}(V)$ ). They satisfy $L^{\text {odd }}(V) \subset L^{\text {even }}(V)$. The subspaces $R^{\text {odd }}(V)$ and $R^{\text {even }}(V)$ are defined similarly and satisfy $R^{\text {odd }}(V) \subset R^{\text {even }}(V)$. With these notations, and assuming that $f \notin \mathcal{N}$, we have

$$
V_{\mathrm{tdeg}}=L^{\text {odd }}(V) \oplus R^{\text {odd }}(V), \quad V_{\text {ndeg }}=L^{\text {even }}(V) \cap R^{\text {even }}(V) .
$$

For $\lambda \in K$ we set $\hat{\lambda}:=\left\{\lambda, \lambda^{-1}\right\}$ if $\lambda \neq 0$ and $\hat{\lambda}:=\{0\}$ if $\lambda=0$. We also set $\hat{K}:=\{\hat{\lambda}: \lambda \in K\}$.

When $f$ is nondegenerate, the asymmetry of $f$ is the unique $\sigma \in \mathrm{GL}_{n}$ such that $f^{\prime}(v, w)=f(v, \sigma(w))$ for all $v, w \in V$. It follows that the matrix of $\sigma$ with respect to the same basis is $A_{f}^{-1} A_{f}^{\prime}$.

We can now refine the previous proposition.

Proposition 3.4. For $f \in \mathcal{B} \backslash \mathcal{N}$ there is a unique decomposition

$$
(V, f)=\perp_{\hat{\lambda} \in \hat{K}}\left(V^{\hat{\lambda}}, f^{\hat{\lambda}}\right)
$$

such that $V_{\mathrm{tdeg}}=V^{\hat{0}}$ and for $\lambda \neq 0,\left(V^{\hat{\lambda}}, f^{\hat{\lambda}}\right)$ is nondegenerate and $\hat{\lambda}$ is the set of eigenvalues of the asymmetry $\sigma^{\hat{\lambda}}$ of $f^{\hat{\lambda}}$. Consequently,

$$
\mathrm{GL}(V)_{f}=\prod_{\hat{\lambda} \in \hat{K}} \mathrm{GL}\left(V^{\hat{\lambda}}\right)_{f^{\hat{\lambda}}}
$$

Proof. We first make use of Proposition 3.3 to decompose $V$ into orthogonal

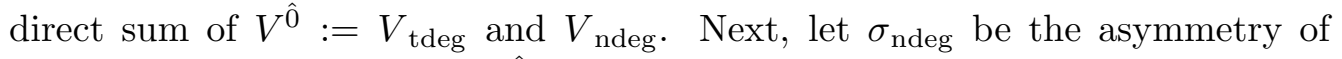
$f_{\text {ndeg. For }} \lambda \neq 0$, we define $V^{\hat{\lambda}}$ to be the sum of the generalized eigenspaces of $\sigma_{\text {ndeg }}$ associated with the eigenvalues $\lambda$ and $\lambda^{-1}$. It is easy to show (or see [9]) that $V^{\hat{\lambda}} \perp V^{\hat{\mu}}$ if $\hat{\lambda} \neq \hat{\mu}$. 
Definition 3.5. For $f \in \mathcal{B} \backslash \mathcal{N}$, we refer to the decomposition (3.3) as the primary decomposition of the bilinear space $(V, f)$. This decomposition will also be written as

$$
V=\perp_{\hat{\lambda} \in \hat{K}} V_{f}^{\hat{\lambda}}
$$

where $V_{f}^{\hat{\lambda}}=V^{\hat{\lambda}}$. The summand $\left(V^{\hat{\lambda}}, f^{\hat{\lambda}}\right)$ is its primary component of type $\hat{\lambda}$. If $V=V^{\hat{\lambda}}$ for some $\hat{\lambda}$, we say that the bilinear form $f$ is $\hat{\lambda}$-primary.

\section{Jordan decomposition of bilinear forms}

In this section we define Jordan decomposition for bilinear forms and discuss some of its properties.

Proposition 4.1. Let $f \in \mathcal{B}$ be semisimple. Then $\left(\mathrm{GL}_{n}\right)_{f}$ and $\left(\mathrm{SL}_{n}\right)_{f}$ are reductive groups. If $f \neq 0$, these groups have the same dimension.

Proof. Since $f$ is semisimple, $\mathcal{O}_{f}$ is closed in $\mathcal{B}$. Hence this orbit is an affine variety. Since the homogeneous space $\mathrm{SL}_{n} /\left(\mathrm{SL}_{n}\right)_{f}$ is isomorphic to $\mathcal{O}_{f}$ (as an algebraic variety with $\mathrm{SL}_{n}$-action, see e.g. [8, p. 152]), this homogeneous space is also an affine variety. By Matsushima's criterion [8, Theorem 4.17], $\left(\mathrm{SL}_{n}\right)_{f}$ is a reductive group. As

$$
\mathrm{GL}_{n} \cdot f=\left\{\lambda g: g \in \mathcal{O}_{f}, \lambda \in K^{*}\right\},
$$

we have $\operatorname{dim}\left(\mathrm{GL}_{n} \cdot f\right)=\operatorname{dim}\left(\mathrm{SL}_{n} \cdot f\right)+1$. Since $\operatorname{dim} \mathrm{GL}_{n}=\operatorname{dim} \mathrm{SL}_{n}+1$, we infer that $\left(\mathrm{GL}_{n}\right)_{f}$ and $\left(\mathrm{SL}_{n}\right)_{f}$ have the same dimension. Consequently, they share the same identity component and so $\left(\mathrm{GL}_{n}\right)_{f}$ is reductive too.

For brevity, we denote the stabilizer of $f$ in $\mathrm{GL}_{n}$ by $Z(f)$, i.e.,

$$
Z(f)=\left(\mathrm{GL}_{n}\right)_{f}=\left\{a \in \mathrm{GL}_{n}: a \cdot f=f\right\} .
$$

The tangent space of $\mathcal{O}_{f}$ at the point $f$ will be denoted by $T_{f}$. Note that

$$
T_{f}=\mathfrak{s l}_{n} \cdot f=\left\{x \cdot f: x \in \mathfrak{s l}_{n}\right\} .
$$

Let $g \in \mathcal{B}$ be semisimple. By the above proposition, $Z(g)$ is a reductive subgroup of $\mathrm{GL}_{n}$. The tangent space $T_{g}$ is $Z(g)$-invariant. Since $Z(g)$ is reductive, $\mathcal{B}=T_{g} \oplus N$ where $N$ is a $Z(g)$-invariant subspace. We denote by $\mathcal{N}_{g}$ the null-cone of the induced action of $\left(\mathrm{SL}_{n}\right)_{g}=Z(g) \cap \mathrm{SL}_{n}$ on $N$.

We shall denote by $N^{\#}$ the largest $\left(\mathrm{SL}_{n}\right)_{g}$-submodule of $N$ which contains no 1-dimensional trivial submodules. This submodule is important because $\mathcal{N}_{g} \subset$ $N^{\#}$. To prove the last assertion, observe that $N=N_{1} \oplus N^{\#}$, where $N_{1}$ is the trivial $\left(\mathrm{SL}_{n}\right)_{g}$-module, and that every $\left(\mathrm{SL}_{n}\right)_{g}$-orbit in $\mathcal{N}_{g}$ must contain 0 in its closure.

Now we can define Jordan decomposition for bilinear forms.

Definition 4.2. For $f \in \mathcal{B}$, we say that the decomposition

$$
f=f_{\mathrm{ss}}+f_{\mathrm{nl}}
$$

is a Jordan decomposition of $f$ if 
(J1) $f_{\mathrm{ss}} \in \mathcal{C}_{f}$

(J2) $f_{\mathrm{nl}} \in \mathcal{N}_{f_{\mathrm{ss}}}$ (for some choice of a $Z\left(f_{\mathrm{ss}}\right)$-invariant complement $N$ of $T_{f_{\mathrm{ss}}}$ in $\mathcal{B})$; and

(J3) $Z(f)=Z\left(f_{\mathrm{ss}}\right) \cap Z\left(f_{\mathrm{nl}}\right)$.

It is immediate from this definition that if (4.1) is a Jordan decomposition of $f$ and $a \in \mathrm{GL}_{n}$, then $a \cdot f=a \cdot f_{\mathrm{ss}}+a \cdot f_{\mathrm{nl}}$ is a Jordan decomposition of $a \cdot f$.

Note that if $f \in \mathcal{B}$ is semisimple (resp. a null-form) then it has a Jordan decomposition (4.1) with $f_{\mathrm{ss}}=f, f_{\mathrm{nl}}=0$ (resp. $\left.f_{\mathrm{ss}}=0, f_{\mathrm{nl}}=f\right)$. In fact if $f$ is a null-form this is the only Jordan decomposition of $f$ because in that case $\mathcal{C}_{f}=\{0\}$. As we will see later, the analogous assertion is valid when $f$ is semisimple.

In the following lemma we show that $f_{\mathrm{ss}}$ and $f_{\mathrm{nl}}$ preserve the orthogonal decompositions with respect to $f$.

Lemma 4.3. Let $f \in \mathcal{B} \backslash \mathcal{N}$ and let (4.1) be a Jordan decomposition of $f$. If

$$
(V, f)=\left(V_{1}, f_{1}\right) \perp\left(V_{2}, f_{2}\right),
$$

then $V_{1}$ and $V_{2}$ are orthogonal to each other with respect to $f_{\mathrm{ss}}$ and $f_{\mathrm{nl}}$.

Proof. Let $a \in \mathrm{GL}_{n}$ be the involution which is 1 on $V_{1}$ and -1 on $V_{2}$. The assertion now follows from the fact that $a \in Z(f)=Z\left(f_{\mathrm{ss}}\right) \cap Z\left(f_{\mathrm{nl}}\right)$.

Assume that $f \in \mathcal{B} \backslash \mathcal{N}$ and consider its primary decomposition (3.3).

For $\hat{\lambda}, \hat{\mu} \in \hat{K}$ we denote by $\mathcal{B}(f ; \hat{\lambda}, \hat{\mu})$ the subspace of $\mathcal{B}$ consisting of all forms $g$ such that $g\left(V^{\hat{\xi}}, V^{\hat{\eta}}\right)=0$ if $\hat{\xi} \neq \hat{\lambda}$ or $\hat{\eta} \neq \hat{\mu}$.

We have $\mathcal{B}=\mathcal{B}_{f} \oplus \mathcal{B}^{f}$ where

$$
\mathcal{B}_{f}:=\bigoplus_{\hat{\lambda} \in \hat{K}} \mathcal{B}(f ; \hat{\lambda}, \hat{\lambda})
$$

and

$$
\mathcal{B}^{f}:=\bigoplus_{\hat{\lambda} \neq \hat{\mu}} \mathcal{B}(f ; \hat{\lambda}, \hat{\mu})
$$

Lemma 4.4. If $f \in \mathcal{B} \backslash \mathcal{N}$, then $\mathcal{B}^{f} \subset T_{f}$.

Proof. It suffices to show that $\mathcal{B}(f ; \hat{\lambda}, \hat{\mu})+\mathcal{B}(f ; \hat{\mu}, \hat{\lambda}) \subset T_{f}$ for $\hat{\lambda} \neq \hat{\mu}$. Thus we may assume that $V=V^{\hat{\lambda}} \oplus V^{\hat{\mu}}$. We may also assume that our basis $\alpha$ of $V$ is chosen as the union of bases of $V^{\hat{\lambda}}$ and $V^{\hat{\mu}}$. Then the matrix of $f$ has the form $A=B \oplus C$, where $B$ resp. $C$ is the matrix of $f^{\hat{\lambda}}$ resp. $f^{\hat{\mu}}$. Let us take $X \in \mathfrak{s l}_{n}$ of the form

$$
X=\left[\begin{array}{ll}
0 & Y \\
Z & 0
\end{array}\right]
$$


Then the bilinear form $-X \cdot f$ belongs to the tangent space $T_{f}$ and is given by the matrix

$$
X^{\prime} A+A X=\left[\begin{array}{cc}
0 & B Y+Z^{\prime} C \\
C Z+Y^{\prime} B & 0
\end{array}\right] .
$$

We complete the proof by an obvious dimension argument: It suffices to show that the matrix equations

$$
B Y+Z^{\prime} C=0, \quad C Z+Y^{\prime} B=0
$$

have only the zero solution, i.e., that they imply $Y=Z=0$. Without any loss of generality, we may assume that $\mu \neq 0$, i.e., that $C$ is invertible. Then $Z=-C^{-1} Y^{\prime} B$ and it remains to show that the equation $B Y C^{-1} C^{\prime}=B^{\prime} Y$ implies $Y=0$. This is indeed so if also $\lambda \neq 0$ since the asymmetries of $f^{\hat{\lambda}}$ and $f^{\hat{\mu}}$, with matrices $S_{\lambda}:=B^{-1} B^{\prime}$ and $S_{\mu}:=C^{-1} C^{\prime}$ respectively, have no common eigenvalues.

Assume now that $\lambda=0$. By using a suitable basis of $V^{\hat{\lambda}}$,

$$
B=\left[\begin{array}{cc}
0 & I_{r} \\
J & 0
\end{array}\right]
$$

where $J$ is an $r$-by- $r$ nilpotent matrix. Let us partition $Y$ into two blocks: The top block $Y_{1}$ and the bottom block $Y_{2}$, each having $r$ rows. Then the equation $B Y S_{\mu}=B^{\prime} Y$ gives $Y_{1}=J Y_{1} S_{\mu}$ and $Y_{2} S_{\mu}=J^{\prime} Y_{2}$. Thus $Y_{1}=J Y_{1} S_{\mu}=$ $J^{2} Y_{1} S_{\mu}^{2}=\cdots$, so $Y_{1}=0$. Similarly, $Y_{2}=0$. Hence the lemma is proved.

We now show that the construction of Jordan decompositions can be reduced to the primary case.

Theorem 4.5. Let $f=g+h$ be a Jordan decomposition ( $g$ semisimple, $h a$ null-form) of $f \in \mathcal{B} \backslash \mathcal{N}$. Then the primary decompositions of $f$ and $g$ have the same underlying orthogonal direct vector space decomposition

$$
V=\perp_{\hat{\lambda} \in \hat{K}} V^{\hat{\lambda}}
$$

Moreover, the restrictions $f^{\hat{\lambda}}, g^{\hat{\lambda}}$ and $h^{\hat{\lambda}}$ of $f, g$ and $h$, respectively, to $V^{\hat{\lambda}} \times V^{\hat{\lambda}}$ give a Jordan decomposition $f^{\hat{\lambda}}=g^{\hat{\lambda}}+h^{\hat{\lambda}}$.

Proof. Let us assume that the primary components of $(V, g)$ are $\left(V^{\hat{\lambda}}, g^{\hat{\lambda}}\right)$.

By Proposition 3.4 we have

$$
Z(g)=\prod_{\hat{\lambda} \in \hat{K}} Z\left(g^{\hat{\lambda}}\right) .
$$

Denote by $E_{g}$ the elementary abelian 2-subgroup of $Z(g)$ consisting of all operators which leave each $V_{g}^{\hat{\lambda}}$ invariant and act on it as \pm 1 .

Let $N$ be the $Z(g)$-invariant complement of $T_{g}$ used in the construction of Jordan decomposition $f=g+h$. Since $N$ is invariant under the action of $E_{g}$, $N$ must be the direct sum of its intersections with the subspaces $\mathcal{B}(g ; \hat{\lambda}, \hat{\mu})+$ 
$\mathcal{B}(g ; \hat{\mu}, \hat{\lambda})$. By Lemma 4.4 we have $\mathcal{B}^{g} \subset T_{g}$, and since $N \cap T_{g}=0$ we conclude that

$$
N=\bigoplus_{\hat{\lambda}} N \cap \mathcal{B}(g, \hat{\lambda}, \hat{\lambda})
$$

Since a nontrivial simple $Z(g)$-submodule of $\mathcal{B}(g ; \hat{\lambda}, \hat{\lambda})$ cannot be isomorphic to a nontrivial simple $Z(g)$-submodule of $\mathcal{B}(g ; \hat{\mu}, \hat{\mu})$ if $\hat{\lambda} \neq \hat{\mu}$, we also have

$$
N^{\#}=\bigoplus_{\hat{\lambda}} N^{\#} \cap \mathcal{B}(g, \hat{\lambda}, \hat{\lambda}) .
$$

Now, the fact that $h \in \mathcal{N}_{g} \subset N^{\#}$ implies that the primary decomposition with respect to $g$ is also orthogonal with respect to $h$ (and consequently $f$ ). We can now define $f^{\hat{\lambda}}$ and $h^{\hat{\lambda}}$ to be the restrictions of $f$ and $h$, respectively, to $V^{\hat{\lambda}} \times V^{\hat{\lambda}}$.

Note that $h^{\hat{\lambda}} \in N^{\#} \cap \mathcal{B}(g ; \hat{\lambda}, \hat{\lambda})$ and that, by Proposition 3.2, each $g^{\hat{\lambda}}$ is semisimple. Hence, $Z\left(g^{\hat{\lambda}}\right)$ is reductive and we can choose $Z\left(g^{\hat{\lambda}}\right)$-invariant subspace $N_{\hat{\lambda}} \supset N^{\#} \cap \mathcal{B}(g ; \hat{\lambda}, \hat{\lambda})$ such that

$$
\mathcal{B}(g ; \hat{\lambda}, \hat{\lambda})=T_{g_{\hat{\lambda}}} \oplus N_{\hat{\lambda}}
$$

By the Hilbert-Mumford criterion (see [8, Theorem 5.2]) there exists a 1dimensional torus $X(t) \subset Z(g)$ such that $0 \in \overline{X(t) \cdot h}$. If $X_{\hat{\lambda}}(t)$ is the image of $X(t)$ under the projection $Z(g) \rightarrow Z\left(g^{\hat{\lambda}}\right)$, then $X_{\hat{\lambda}}(t) \cdot g^{\hat{\lambda}}=g^{\hat{\lambda}}$. It follows that $X_{\hat{\lambda}}(t) \in \operatorname{SL}\left(V^{\hat{\lambda}}\right)$. Since $0 \in \overline{X_{\hat{\lambda}}(t) \cdot h^{\hat{\lambda}}}$, we conclude that $h^{\hat{\lambda}} \in \mathcal{N}_{g^{\hat{\lambda}}}$ and, consequently, $g^{\hat{\lambda}} \in \mathcal{C}_{f \hat{\lambda}}$.

It is now easy to verify that $f^{\hat{\lambda}}=g^{\hat{\lambda}}+h^{\hat{\lambda}}$ is a Jordan decomposition of $f^{\hat{\lambda}}$. Hence, $V_{g}^{\hat{\lambda}}=V_{f}^{\hat{\lambda}}$ for each $\hat{\lambda}$ and the proof is completed.

We shall now prove the converse.

Theorem 4.6. Let (3.3) be the primary decomposition of $f \in \mathcal{B} \backslash \mathcal{N}$. If $f^{\hat{\lambda}}=$ $g^{\hat{\lambda}}+h^{\hat{\lambda}}, \hat{\lambda} \in \hat{K}$, are Jordan decompositions ( $g^{\hat{\lambda}}$ semisimple, $h^{\hat{\lambda}}$ a null-form), then

$$
g:=\perp_{\hat{\lambda} \in \hat{K}} g^{\hat{\lambda}} \quad \text { and } \quad h:=\perp_{\hat{\lambda} \in \hat{K}} h^{\hat{\lambda}}
$$

give a Jordan decomposition of $f$.

Proof. By Proposition 3.2, $g$ is semisimple. By Proposition 3.4, $Z(g)$ is the direct product of the groups $Z\left(g^{\hat{\lambda}}\right), \hat{\lambda} \in \hat{K}$.

Let $\mathcal{B}(f, \hat{\lambda}, \hat{\lambda})=T_{g^{\hat{\lambda}}} \oplus N_{g^{\hat{\lambda}}}$ be the decomposition of $Z\left(g^{\hat{\lambda}}\right)$-modules such that $h^{\hat{\lambda}} \in \mathcal{N}_{g^{\hat{\lambda}}}$. By using Lemma 4.4 and the fact that $Z(g)$ is reductive, we can choose a direct decomposition $\mathcal{B}=T_{g} \oplus N$ of $Z(g)$-modules such that $N \supset N_{g^{\hat{\lambda}}}^{\#}$ for each $\hat{\lambda}$. Thus $h \in \mathcal{N}_{g}$, proving that $f=g+h$ is a Jordan decomposition. 
We can now prove the uniqueness of Jordan decomposition for semisimple bilinear forms.

Proposition 4.7. If $f \in \mathcal{B}$ is semisimple, it has only one Jordan decomposition: $f_{\mathrm{ss}}=f, f_{\mathrm{nl}}=0$.

Proof. We may assume that $f \neq 0$ and so $f \notin \mathcal{N}$. Let (4.1) be a Jordan decomposition of $f$. We have to show that $f_{\mathrm{ss}}=f$. In view of Theorem 4.5, it suffices to consider the primary case, i.e., we may assume that the primary decomposition (3.4) reduces to $V=V^{\hat{\lambda}}$ for some $\hat{\lambda} \in \hat{K}$.

Assume first that $\lambda \neq \pm 1$. Since $f$ is semisimple, Proposition 3.2 implies that there exists a basis of $V$ such that

$$
f=\left[\begin{array}{cc}
0 & I_{m} \\
\lambda I_{m} & 0
\end{array}\right] .
$$

If $a:=b^{\prime} \oplus b^{-1}$, where $b \in \mathrm{GL}_{m}$, then $a \in Z(f)$. By (J3) we also have $a \in Z\left(f_{\mathrm{ss}}\right)$. It follows that

$$
f_{\mathrm{ss}}=\left[\begin{array}{cc}
0 & \mu I_{m} \\
\nu I_{m} & 0
\end{array}\right]
$$

for some $\mu, \nu \in K$. As $f_{\mathrm{nl}}=f-f_{\mathrm{ss}} \in \mathcal{N}$, we must have $\mu=1$ and $\nu=\lambda$, i.e., $f_{\mathrm{ss}}=f$.

Next assume that $\lambda=1$. Since $f$ is semisimple, its asymmetry $\sigma=1$, i.e., $f$ is symmetric. By using Proposition 3.2, we conclude that we can choose a basis of $V$ such that $f=\mu I_{n}$ for some $\mu \in K^{*}$. Hence $Z(f)$ is the orthogonal group. As $Z(f) \subset Z\left(f_{\mathrm{ss}}\right)$, it follows that also $f_{\mathrm{ss}}=\nu I_{n}$ for some $\nu \in K^{*}$. As $f_{\mathrm{nl}}=f-f_{\mathrm{ss}} \in \mathcal{N}$, we must have $\nu=\mu$, i.e., $f_{\mathrm{ss}}=f$.

The case $\lambda=-1$ is similar to $\lambda=1$.

\section{Jordan decomposition in the primary case}

In view of Theorem 4.5 and 4.6, the construction of Jordan decomposition for bilinear forms $f \in \mathcal{B} \backslash \mathcal{N}$ has been reduced to the primary case. In this section we shall assume that $f$ is $\hat{\lambda}$-primary.

We start with the case $\lambda \neq \pm 1$. Then the indecomposable components of $f$ are necessarily of type (b1) (see Theorem 2.1). Consequently, $n=2 m$ is even.

It is easy to check that $f-\lambda f^{\prime} \notin \mathcal{N}$ and is $\hat{0}$-primary. Let $V_{1}:=R^{\text {odd }}(V)$ and $V_{2}:=L^{\text {odd }}(V)$ with respect to $f-\lambda f^{\prime}$. These subspaces are totally isotropic for both $f$ and $f-\lambda f^{\prime}$. They have the same dimension and $V=V_{1} \oplus V_{2}$. For instance, if $f$ is given by the matrix $H_{n}(\lambda)$, then $V_{1}$ resp. $V_{2}$ is spanned by the last resp. first $m$ basis vectors of $V$.

We remark that if also $\lambda \neq 0$, then $V_{1}$ resp. $V_{2}$ is the generalized eigenspace of the asymmetry $\sigma$ of $f$ for the eigenvalue $1 / \lambda$ resp. $\lambda$.

Let $\mu \in K \cup\{\infty\}, \mu \neq \lambda$. We define bilinear forms $g_{\mu}, h_{\mu} \in \mathcal{B}$ via "matrices":

$$
g_{\mu}=\left[\begin{array}{cc}
0 & \frac{f^{\prime}-\mu f}{\lambda-\mu} \\
\lambda \cdot \frac{f-\mu f^{\prime}}{\lambda-\mu} & 0
\end{array}\right], \quad h_{\mu}=\left[\begin{array}{cc}
0 & \frac{\lambda f-f^{\prime}}{\lambda-\mu} \\
\mu \cdot \frac{\lambda f^{\prime}-f}{\lambda-\mu} & 0
\end{array}\right] .
$$


More precisely this means that the subspaces $V_{1}$ and $V_{2}$ are totally isotropic with respect to $g_{\mu}$, the restriction of $g_{\mu}$ to $V_{1} \times V_{2}$ coincides with that of $\left(f^{\prime}-\mu f\right) /(\lambda-$ $\mu$ ), and the restriction of $g_{\mu}$ to $V_{2} \times V_{1}$ coincides with that of $\lambda\left(f-\mu f^{\prime}\right) /(\lambda-\mu)$. The interpretation of the definition of $h_{\mu}$ is similar. If $\mu=\infty$ then one should interprete the above "matrix" entries in the usual way, e.g. $\left(f^{\prime}-\mu f\right) /(\lambda-\mu)=f$.

We shall prove below that $g_{\mu}$ is semisimple, that $h_{\mu}$ is a null-form, and that $f=g_{\mu}+h_{\mu}$ is a Jordan decomposition.

Theorem 5.1. Let $f \in \mathcal{B} \backslash \mathcal{N}$ be a $\hat{\lambda}$-primary bilinear form with $\lambda \neq \pm 1$. Then for $\mu \in K \cup\{\infty\}$ and $\mu \neq \lambda$, we have a Jordan decomposition $f=g_{\mu}+h_{\mu}$ where $g_{\mu}$ and $h_{\mu}$ are as defined above. Moreover, these exhaust all possible Jordan decompositions of $f$.

Proof. Let $f=g+h$ be an arbitrary Jordan decomposition with $g$ semisimple and $h$ a null-form. By the Canonical Form Theorem, each indecomposable summand of $(V, f)$ is of type (b1), i.e., its matrix in a suitable basis is $H_{2 k}(\lambda)$ for some positive integer $k$. It follows that, with respect to a suitable basis of $V$, the matrix of $f$ will be

$$
f=\left[\begin{array}{cc}
0 & I_{m} \\
\lambda I_{m}+J & 0
\end{array}\right]
$$

where $J$ is a nilpotent matrix in its lower triangular Jordan canonical form.

The stabilizer $Z(f)$ also fixes the form $f^{\prime}$ and the form $\varphi:=f-\lambda f^{\prime}$. As $V_{1}=R_{\varphi}^{\text {odd }}(V)$ and $V_{2}=L_{\varphi}^{\text {odd }}(V), V_{1}$ and $V_{2}$ are $Z(f)$-invariant subspaces. Now an easy computation shows that

$$
Z(f)=\left\{\left[\begin{array}{cc}
A & 0 \\
0 & \left(A^{\prime}\right)^{-1}
\end{array}\right]: A \in \mathrm{GL}_{m}, A J=J A\right\} .
$$

By (J3), $Z(f)$ fixes $g$. Hence we must have

$$
g=\left[\begin{array}{cc}
0 & X \\
Y & 0
\end{array}\right]
$$

Furthermore, a computation shows that $A^{\prime} X=X A^{\prime}$ and $A Y=Y A$ for all $A \in \mathrm{GL}_{m}$ such that $A J=J A$. We conclude that $X=\varphi(J)^{\prime}$ and $Y=\psi(J)$ for some $\varphi(t), \psi(t) \in K[t]$.

Since $h \in \mathcal{N}$, we deduce that $\varphi(0)=1$ and $\psi(0)=\lambda$. Hence

$$
\varphi(t)=1-t \varphi_{0}(t), \quad \psi(t)=\lambda\left(1-t \psi_{0}(t)\right),
$$

for some polynomials $\varphi_{0}(t)$ and $\psi_{0}(t)$. We now see that the matrix of $g$ is congruent to

$$
\left[\begin{array}{cc}
0 & I_{m} \\
\lambda\left(I_{m}-J \psi_{0}(J)\right)\left(I_{m}-J \varphi_{0}(J)\right)^{-1} & 0
\end{array}\right] .
$$

It is immediate from Proposition 3.2 that, since $g$ is semisimple and nondegenerate, its asymmetry has to be semisimple. We conclude that $J \psi_{0}(J)=J \varphi_{0}(J)$. 
The matrices of $g$ and $h$ are given by:

$g=\left[\begin{array}{cc}0 & I_{m}-J^{\prime} \varphi_{0}\left(J^{\prime}\right) \\ \lambda\left(I_{m}-J \varphi_{0}(J)\right) & 0\end{array}\right], h=\left[\begin{array}{cc}0 & J^{\prime} \varphi_{0}\left(J^{\prime}\right) \\ J\left(I_{m}+\lambda \varphi_{0}(J)\right) & 0\end{array}\right]$.

If $f$ is semisimple, then $J=0$ and so $g=f$ and $h=0$ (which agrees with Proposition 4.7). Hence, we may assume that $f$ is not semisimple, i.e., $J \neq 0$.

For the sake of brevity set $P:=I_{m}-J \varphi_{0}(J)$. Further computations show that

$$
T_{g}=\left\{\left[\begin{array}{cc}
x & y^{\prime} P^{\prime} \\
\lambda P y & z
\end{array}\right]: y \in \mathfrak{s l}_{m}, x, z \in \mathfrak{g l}_{m}\right\}
$$

and

$$
Z(g)=\left\{\left[\begin{array}{cc}
P^{-1} x^{\prime} P & 0 \\
0 & x^{-1}
\end{array}\right]: x \in \mathrm{GL}_{m}\right\} .
$$

If $N$ is a $Z(g)$-invariant complement of $T_{g}$ in $\mathcal{B}$, then $N$ is a direct sum of three simple $Z(g)$-modules, exactly two of which are 1-dimensional. Hence, we must have

$$
N^{\#}=\left\{\left[\begin{array}{cc}
0 & y^{\prime} P^{\prime} \\
\mu P y & 0
\end{array}\right]: y \in \mathfrak{s l}_{m}\right\}, \quad \mu \in K,
$$

for some fixed $\mu \neq \lambda$ or

$$
N^{\#}=\left\{\left[\begin{array}{cc}
0 & 0 \\
P y & 0
\end{array}\right]: y \in \mathfrak{s l}_{m}\right\}
$$

in which case we set $\mu=\infty$.

Assuming that $\mu \neq \infty$, from $h \in \mathcal{N}_{g} \subset N^{\#}$ we obtain that $J\left(I_{m}+\lambda \varphi_{0}(J)\right)=$ $\mu J \varphi_{0}(J)$, i.e.,

$$
J\left(I_{m}+(\lambda-\mu) \varphi_{0}(J)\right)=0 .
$$

Since $J$ is nilpotent and nonzero, this equation implies that $\varphi_{0}(J)=(\mu-\lambda)^{-1} I_{m}$. Hence

$g=\left[\begin{array}{cc}0 & I_{m}+(\lambda-\mu)^{-1} J^{\prime} \\ \lambda\left(I_{m}+(\lambda-\mu)^{-1} J\right) & 0\end{array}\right], h=(\mu-\lambda)^{-1} \cdot\left[\begin{array}{cc}0 & J^{\prime} \\ \mu J & 0\end{array}\right]$.

It is clear that these $g$ and $h$ indeed provide a Jordan decomposition of $f$.

By comparing with (5.1), we see that $g=g_{\mu}$ and $h=h_{\mu}$.

The case $\mu=\infty$ can be treated similarly.

We remark that if $f$ in the above proposition is not semisimple, then the nullform component $h_{\mu}$ is symmetric resp. skew-symmetric iff $\mu=1$ resp. $\mu=-1$.

Now let us assume that $\lambda= \pm 1$. In this case we have the following, somewhat unexpected and surprising, result.

Theorem 5.2. Let $f \in \mathcal{B} \backslash \mathcal{N}$ be a $\hat{\lambda}$-primary bilinear form with $\lambda= \pm 1$. Then $f$ has precisely one Jordan decomposition $f=f_{\mathrm{ss}}+f_{\mathrm{nl}}$. It is given by $f_{\mathrm{ss}}=f^{+}$, $f_{\mathrm{nl}}=f^{-}$if $\lambda=1$ and by $f_{\mathrm{ss}}=f^{-}, f_{\mathrm{nl}}=f^{+}$if $\lambda=-1$. 
Proof. We shall give the proof for the case $\lambda=1$, the other case being similar.

We have to verify that $f=f^{+}+f^{-}$is indeed a Jordan decomposition. The conditions (J1) and (J3) are clearly satisfied. The condition (J2) follows from the proof of Proposition 3.1, the fact that $T_{f} \subset \mathcal{B}^{+}$, and that we can choose $N$ to contain $\mathcal{B}^{-}$. It is easy to verify that the decomposition given in the theorem is indeed a Jordan decomposition. We leave this verification to the reader.

Let $f=g+h$ be a Jordan decomposition of $f$ with $g$ semisimple and $h$ a null-form. As $f$ is nondegenerate, so is $g$. Since $\lambda=1$, the indecomposable summands of $f$ are of type $H_{k}(1)$ with $k$ divisible by 4 or $\Gamma_{k}$ for odd $k$. It follows from Proposition 3.1 that $\mathcal{C}_{f} \subset \mathcal{B}^{+}$. Consequently, $g \in \mathcal{B}^{+}$and we conclude that $Z(g)=\mathrm{O}(g)$ and $\left(\mathrm{SL}_{n}\right)_{g}=\mathrm{SO}(g)$. Hence, $T_{g}$ is a hyperplane in $\mathcal{B}^{+}$. More precisely,

$$
T_{g}=\left\{\varphi \in \mathcal{B}^{+}: \operatorname{tr}\left(A_{g}^{-1} A_{\varphi}\right)=0\right\},
$$

and we have $\mathcal{B}^{+}=K g \oplus T_{g}$. Note that $T_{g}$ and $\mathcal{B}^{-}$are simple but non-isomorphic $Z(g)$-modules. It follows that if $N$ is any $Z(g)$-invariant subspace of $\mathcal{B}$ such that $\mathcal{B}=T_{g} \oplus N$, then $N^{\#} \subset \mathcal{B}^{-}$. Therefore $h \in \mathcal{N}_{g} \subset N^{\#}$ shows that $h \in \mathcal{B}^{-}$. It follows that $g=f^{+}$and $h=f^{-}$.

The following corollary follows from Theorems 4.5, 5.1 and 5.2.

Corollary 5.3. Let $(V, f)=\left(V_{1}, f_{1}\right) \perp\left(V_{2}, f_{2}\right)$ and let $f=g+h$ be a Jordan decomposition of $f$ with $g=g_{1} \perp g_{2}$ semisimple and $h=h_{1} \perp h_{2}$ a null-form. Then $f_{i}=g_{i}+h_{i}$ is a Jordan decomposition of $f_{i}$.

\section{References}

[1] O.M. Adamovich and E.O. Golovina, On the invariants of a pair of bilinear forms, Vestn. Mosk. Univ., Ser. I, 2 (1977), 15-18.

[2] B. Corbas and G.D. Williams, Bilinear forms over an algebraically closed field, J. Pure Appl. Algebra, 165 (2001), 255-266.

[3] D.Ž. Đoković, J. Sekiguchi, and K. Zhao, On the geometry of unimodular congruence classes of bilinear forms, preprint, 2004.

[4] D.Ž. Đoković and F. Szechtman, Characterization of bilinear forms with unimodular isometry group, Proc. Amer. Math. Soc. (to appear).

[5] P. Gabriel, Appendix: Degenerate bilinear forms, J. Algebra, 31 (1974), 67-72.

[6] R.A. Horn and V.V. Sergeichuk, Congruences of a square matrix and its transpose, Linear Algebra Appl. 389 (2004), 347-353.

[7] V. Gatti and E. Viniberghi, Spinors of 13-dimensional space, Advances in Mathematics, 30 (1978), 137-155.

[8] V.L. Popov and E.B. Vinberg, Invariant Theory, Encyclopaedia of Mathematical Sciences, Algebraic Geometry IV, Springer, New York, 1994.

[9] C. Riehm, The equivalence of bilinear forms, J. Algebra, 31 (1974), 45-66.

[10] N. Spaltenstein, Dégénérescences des formes bilinéaires, J. Algebra 80 (1983), 1-28.

Department of Pure Mathematics, University of Waterloo, Waterloo, Ontario, N2L 3G1, CANADA

E-mail address: djokovic@uwaterloo.ca 
Department of Mathematics, Wilfrid Laurier University, Waterloo, Ontario, Canada N2L 3C5, and Institute of Mathematics, Academy of Mathematics and System Sciences, Chinese Academy of Sciences, Beijing 100080, P.R. China

E-mail address: kzhao@wlu.ca 